\title{
NDGA-P21, a novel derivative of nordihydroguaiaretic acid, inhibits glioma cell proliferation and stemness
}

\author{
Qi-Wen Zhao ${ }^{1,2}$, Yong Lin ${ }^{1,2}$, Chang-Rong Xu ${ }^{1,2}$, Yue-Liang Yao ${ }^{1,2}$, You-Hong Cui ${ }^{1,2}$, Xia Zhang ${ }^{1,2}$ and Xiu-Wu Bian ${ }^{1,2}$
}

Nordihydroguaiaretic acid (NDGA) and its synthetic chiral analog dl-nordihydroguaiaretic acid (Nordy) show collective benefits in anti-tumor, and defending against viral and bacterial infections. Here, we synthetized a new derivative-NDGAP21 based on NDGA structure. Regardless of the structural similarity, NDGA-P21 exhibited stronger capability in suppression of glioblastoma (GBM) cell growth as compared to Nordy. Mechanically, NDGA-P21 is able to arrest cell cycle of GBM cells in G0/G1 phase, and to block cell proliferation sequentially. It is important to note that NDGA-P21 is able to impair the stemness of glioma stem-like cells (GSLCs) via measurement of colony formation and sphere formation. Taken together, the novel NDGA-based compound NDGA-P21 exhibits potential therty -20 apeutic implications through inhibiting proliferation of glioma cells and self-renewal capability of GSLCs.

Laboratory Investigation (2017) 97, 1180-1187; doi:10.1038/labinvest.2017.46; published online 15 May 2017

Cancer has been a worldwide threat to public health, and the leading cause of death, particularly since 2010 in China. ${ }^{1}$ Malignant brain tumors are leading cause for cancer death of children and adolescents, and glioma is one of the most malignant tumors up to now. ${ }^{2}$ Recent decades, people have great progress in improving lifespan and life quality of the patients with such malignant burden, but to our regret, the beneficial effect is still quite limited on glioma. ${ }^{2}$ The need for new drug or other methods to treat glioma is never too much.

Nordihydroguaiaretic acid (NDGA) is a naturel phenolic compound extracted from the leaves of Larrea tridentata (Creosote bush), a kind of evergreen desert shrub. ${ }^{3}$ It is also known as masoprocol, an antineoplastic drug that has benefits as anti-viral remedy, and is used for the treatment of actinic keratosis. However, up to now, it remains elusive how NDGA exactly works. ${ }^{4-6}$ In our previous studies, we synthesized Nordy (dl-NDGA), a chiral NDGA derivative which inhibits the growth of multiple tumors, including glioma. ${ }^{7,8}$ Nordy could also induce differentiation and inhibit self-renewal of glioma stem-like cell (GSLC). ${ }^{9}$

In this paper, we reported to synthesize a novel small molecular compound-NDGA-P21, based on the structure of NDGA. According to several experiments, we found that NDGA-P21 obviously inhibited proliferation of glioma cells and self-renewal of GSLC.

\section{MATERIALS AND METHODS Synthesis and Chemical Structure of 1, 4-Bis (3, 4-Dimethoxy Phenyl)-2, 3-Dimethyl-2, 3-Epoxy Butane (NDGA-P21)}

The substrate (E)-4, 4'-(2, 3-dimethylbut-2-ene-1, 4-diyl) bis (1, 2-dimethoxybenzene) (the detailed synthesis method could be found in Chinese patent: ZL02133700.4.; weighted as $0.87 \mathrm{~g}$ ) was dissolved with $13 \mathrm{ml}$ dichloromethane in a $25 \mathrm{ml}$ round-bottom flask, cooled down to $-10{ }^{\circ} \mathrm{C}$, and was then mixed with $0.90 \mathrm{~g} \mathrm{~m}$-CPBA to stir and react for $2 \mathrm{~h}$. The reaction was then quenched with $4.11 \mathrm{~g} \mathrm{Na}_{2} \mathrm{SO}_{3}$ and stirred continuously for $3 \mathrm{~h}$ at room temperature. The product was extracted with ethyl acetate, washed with saturated $\mathrm{NaCl}$, dried with $\mathrm{Na}_{2} \mathrm{SO}_{4}$ and concentrated to yield rude product $(1.02 \mathrm{~g})$. The rude product was subjected to flash silica gel column chromatography to obtain the pale yellow product $(0.91 \mathrm{~g})$ with the yield of $99.13 \%$.

\section{Identification of Compound}

The HRESIMS of the product gave a peak at $\mathrm{m} / \mathrm{z} 395.1835$ $[\mathrm{M}+\mathrm{Na}]^{+}$, whose molecular weight was $16 \mathrm{Da}$ more than the substrate, indicating an additional oxygen atom was present. Its ${ }^{1} \mathrm{H}$ NMR was almost identical to the substrate's one, except that the chemical shifts of the two methyl at C-2 and C-3 were moved to high field from $\delta \mathrm{H} 1.66$ to $\delta \mathrm{H} 1.31$. Furthermore,

\footnotetext{
${ }^{1}$ Key Laboratory of Tumor Immunopathology, Ministry of Education of China, Southwest Hospital, Third Military Medical University, Chongqing, China and ${ }^{2}$ Institute of Pathology and Southwest Cancer Center, Southwest Hospital, Third Military Medical University, Chongqing, China

Correspondence: Professor X-W Bian, MD, PhD, Institute of Pathology and Southwest Cancer Center, Southwest Hospital, Third Military Medical University, Chongqing 400038, China.
}

E-mail: bianxiuwu@263.net

Received 23 December 2016; revised 4 March 2017; accepted 21 March 2017 
the two olefinic carbon resonances of $\delta \mathrm{C} 133.3$ and 128.6 at C-2 and C-3 were absent, which were replaced by the two overlapped oxygen-carbon resonances of $\delta \mathrm{C} 65.1$ in the ${ }^{13} \mathrm{C}$ NMR spectra of the product. All of these elucidated that the additional oxygen atom was cyclization between C-2 and C-3. The product was named as NDGA-P21.

\section{Cell Culture}

U87-MG cells (ATCC Number: HTB-14) were obtained from ATCC (American Type Culture Collection, Manassas, VA, USA). Primary GBM cells, named GBM1, were separated from a glioblastoma (GBM, WHO Grade IV) patient, and classified as proneural-like subtype owing to the positive staining of PDGFRA and P53, as described in more details previously. ${ }^{10}$ Cells were cultured with Dulbecco's Modified Eagle Medium (DMEM) complete medium, ie, DMEM (Gibco, Mount Waverley, VIC, Australia) containing 10\% FBS (Gibco), $100 \mathrm{U} / \mathrm{ml}$ penicillin $\mathrm{G}$ and $100 \mu \mathrm{g} / \mathrm{ml}$ streptomycin (Gibco). Medium was changed every 1-2 days, and cells were passaged every $2-3$ days. The 3 rd -7 th passaged cells were used for further experiments. U87-MG and primary GBM cells-derived GSLCs were maintained in serum-free neural stem cell culture medium, containing DMEM/F12 (Gibco), B27 supplements (Gibco), human recombinant basic FGF (20 ng/ml; Upstate, Lake Placid, NY, USA), and human recombinant EGF $(20 \mathrm{ng} / \mathrm{ml}$; Sigma-Aldrich, St Louis, MO, USA). Second or more passaged spheres were used in experiments. Sphere cells were dissociated with Accutase (Millipore, Bedford, MA, USA) during passage.

\section{Cell Proliferation Assay}

All compounds were dissolved in DMSO (Sigma-Aldrich) to make stock solution as $100 \mathrm{mmol} / \mathrm{l}$. U87-MG or GBM1 cells were seeded in 24-well plates (Corning, Cambridge, MA, USA), at a concentration of $1 \times 10^{4}$ cells/well. Cells were starved for $12 \mathrm{~h}$ with serum-free DMEM medium, and then treated with $50 \mu \mathrm{mol} / \mathrm{l} \mathrm{NDGA}-\mathrm{P} 21,50 \mu \mathrm{mol} / \mathrm{l}$ Nordy, or $0.5 \%$ o DMSO (drug vehicle). Cells stained with trypan blue were counted with a blood cell counting chamber each day until the indicated endpoint. $\mathrm{IC}_{50}$ of compounds was determined by counting cells with a 24-h-treatment of indicated compounds at concentration between 0 and $100 \mu \mathrm{mol} / \mathrm{l}$. Each treatment was carried out in quadruplicate and the independent experiments were repeated for at least three times.

\section{Apoptosis Assay}

U87-MG cells were seeded in 6-well plates as $2 \times 10^{5}$ cells per well and starved $12 \mathrm{~h}$ before treatment. Then cells were treated with $50 \mu \mathrm{mol} / \mathrm{l}$ NDGA-P21, $50 \mu \mathrm{mol} / \mathrm{l}$ Nordy, or 0.05\% DMSO. Cell apoptosis was tested with an Annexin V kit (eBioscience, San Diego, CA, USA), according to the protocol from manufacture. In brief, the treated U87-MG cells were digested with trypsin, and adjusted as $1 \times 10^{6}$ cells per test. Then the cells were washed with PBS once and the provided $1 \times$ binding buffer once, stained with Annexin
V-APC for 30 min at $4{ }^{\circ} \mathrm{C}$, and washed once with $1 \times$ binding buffer. The sample could stand on ice for $4 \mathrm{~h}$ before flow cytometry analysis. Propidium iodide (PI) was added to sample solution $5 \mathrm{~min}$ before testing with Calibur flow cytometer (BD Biosciences, San Jose, CA, USA). All experimental procedures were carried out gently.

\section{Cell Cycle Analysis}

Administered U87-MG cells and GBM1 cells were starved $12 \mathrm{~h}$ before treatment. Then cells were treated with $25 \mu \mathrm{mol} / \mathrm{l}$ NDGA-P21, $25 \mu \mathrm{mol} / \mathrm{l}$ Nordy, or $0.025 \%$ DMSO. Adherent and non-adherent cells were collected, washed twice with cold PBS, and then fixed in ice-cold $70 \%$ ethanol for $6 \mathrm{~h}$ at $4{ }^{\circ} \mathrm{C}$. Fixed cells were stained with freshly diluted PI staining buffer from a cell cycle and apoptosis kit (Beyotime Biotech, Nantong, China) for $30 \mathrm{~min}$ at $37^{\circ} \mathrm{C}$, and analyzed on a FACS Calibur flow cytometer within $2 \mathrm{~h}$. The resulting histograms were analyzed for cell cycle distribution with ModFit software (Verity Software House, Topsham, ME, USA).

\section{Colony Formation Assay}

Soft agar colony formation assay was carried out by suspending 1000 GSLCs in DMEM complete medium containing 0.35\% noble agar (Difco, BD) and $25 \mu \mathrm{mol} / \mathrm{l}$ NDGA-P21, $25 \mu \mathrm{mol} / \mathrm{l}$ Nordy, or $0.025 \%$ DMSO on top of a solid $0.6 \%$ noble agar DMEM complete medium layer using 6-well plates. Cells were incubated for 14 days and colonies were visualized by using an Olympus SZX-10 fluorescent microscope (Tokyo, Japan). Bright field images were captured with a QImaging digital camera (Surrey, BC, Canada) controlled by Image-Pro Plus 6.0 (Media Cybermetics, Rockville, MD, USA). Colonies with more than 50 cells were calculated under an invert microscope (Olympus CFX40, Tokyo, Japan), and each well was counted on 10 fields. Three independent assays were performed.

\section{Sphere Formation Assay}

Cells were sorted with a BD AriaII flow sorter, and seeded in a 96-well plate at a number of 10 cells per well. Cells cultured in serum-free stem cell culture medium with different treatment for 14 days. Then the formation of spheres was checked in each well, and was calculated for sphere formation rate. Ten wells were set up as a group in each treatment and three independent assays were performed.

\section{Quantitative Real-Time PCR}

Total RNA was extracted from cells treated with $25 \mu \mathrm{mol} / 1$ NDGA-P21, $25 \mu \mathrm{mol} / \mathrm{l}$ Nordy, or $0.025 \%$ DMSO by using RNAiso reagent (Takara, Shiga, Japan), according to TAKARA protocol book. Reverse transcription reaction was performed to transcript mRNA into cDNA with a PrimeScript RT reagent Kit (Takara) on C1000 Thermal Cycler. Real-time PCR was carried out to detect the expression of mRNAs in multiple groups by using SYBR Premix Ex Taq II (Takara) on a CFX96 Real-Time PCR Detection System (Bio-Rad, Hercules, CA, USA). Primers were selected by NCBI primer 
BLAST (https://www.ncbi.nlm.nih.gov/tools/primer-blast/ index.cgi). The sequences for the primers of target gene are: GAPDH-Forward 5'-CAAGCTCATTTCCTGGTATGAC-3'; GAPDH-Reverse $\quad 5^{\prime}$-CAGTGAGGGTCTCTCTCTTCCT-3'; CCNE1-Forward 5'-GCCAGCCTTGGGACAATAATG-3'; CCNE1-Reverse $\quad 5^{\prime}$-CTTGCACGTTGAGTTTGGGT-3'; CDK2-Forward $\quad 5^{\prime}$-CCAGGAGTTACTTCTATGCCTGA-3'; CDK2-Reverse CDK4-Forword CDK4-Reverse CDK6-Forward CDK6-Reverse 5'-TTCATCCAGGGGAGGTACAAC-3'; 5'-ATGGCTACCTCTCGATATGAGC-3'; 5'-CATTGGGGACTCTCACACTCT-3'; 5'-CCAGATGGCTCTAACCTCAGT-3'; 5'-AACTTCCACGAAAAAGAGGCTT-3'; CCNA2-Forward 5'-AGCTATGAGTAAGACTGGCATCC-3'; CCNA2-Reverse $\quad 5^{\prime}$-TGGTTTTACTCTCATCTTGCCAC-3'; CCNB1-Forward 5'-TGCCACGAACAGGCCAATAA-3'; CCNB1-Reverse 5'-GAGAAGCAGAACACCGGAGG-3'; CDC25C-Forward 5'-TCAAGTCTTCGCCTGTGTCC-3'; CDC25C-Reverse $\quad 5^{\prime}$-CTGAGCCAGAGCTTCCTTCC-3'; CDK1-Forword $\quad 5^{\prime}$-TTTCTTTCGCGCTCTAGCCA-3'; CDK1-Reverse 5'-CAATCGGGTAGCCCGTAGAC-3'; CDKN1A-Forword $\quad 5^{\prime}$-AGTCAGTTCCTTGTGGAGCC-3'; CDKN1A-Reverse $\quad 5^{\prime}$-CATTAGCGCATCACAGTCGC-3'; GADD45A-Forword 5'-GAATTCTCGGCTGGAGAGCA-3'; GADD45A-Reverse $\quad 5^{\prime}$-CCACATCTCTGTCGTCGTCC-3'; Sox2-Forward

Sox2-Reverse 5'-CCGTTCATCGACGAGGCTAA-3'; 5'-TAACTGTCCATGCGCTGGTT-3'; 5'-GCAGCGACTATGCACAACGA-3'; 5'-CCAGAGTGGTGACGGAGACA-3'; Oct4-Reverse Nanog-Forward 5'-GAGATGCCTCACACGGAGAC-3'; and Nanog-Reverse 5'-GGGTTGTTTGCCTTTGGGAC-3'.

\section{Immunofluorescence}

In a 24-well plate, U87-MG sphere cells were adhered to a $1 \mathrm{~cm} \times 1 \mathrm{~cm}$ cover-slip coated with poly-D-lysin. GBM1 sphere cells were seeded in poly-D-lysin pre-treated glass bottom cell culture dish (Nest, Wuxi, China). After treatment with $25 \mu \mathrm{mol} / \mathrm{l}$ NDGA-P2 1 or $25 \mu \mathrm{mol} / \mathrm{l}$ Nordy for $48 \mathrm{~h}$, cells were washed three times with PBS, and then fixed in $4 \%$ paraformaldehyde for $20 \mathrm{~min}$ at room temperature. For permeabilization, cells were incubated with PBS containing $0.2 \%$ Triton X-100 for $10 \mathrm{~min}$ at room temperature. After airdry, cells were washed three times with PBS, blocked with 5\% goat serum for 30 minutes at $4{ }^{\circ} \mathrm{C}$, then stained with mouseanti-human GFAP antibody (Boster, Wuhan, China) overnight at $4{ }^{\circ} \mathrm{C}$, rinsed three times with PBS, followed by incubation with second antibody Goat anti-mouse Alexa Fluor 488 (Molecular Probes, Eugene, OR, USA) for $30 \mathrm{~min}$ at $37^{\circ} \mathrm{C}$, washed twice with PBS and once with distilled water. Finally, the cover-slip was mounted to U87-MG cells with a drop of prolong gold anti-fade reagent with DAPI (Molecular Probes). GBM1 cells in dish were stained with DAPI (Molecular Probes) for $10 \mathrm{~min}$ at room temperature in dark. Fluorescent images were captured by a fluorescent microscope (Leica DM3000, Germany) or a laser confocal microscope (Zeiss LSM780, Germany).

\section{Statistical Analysis}

All experiments were repeated at least three times and each treatment was set up in triplicate, unless specially indicated otherwise. Data were presented as mean \pm s.e.m. The statistical significance of difference among groups was obtained by the Student's $t$ test or two-way ANOVA multiple comparisons with GraphPad Prism 6 (GraphPad, San Diego, California). All comparisons with a $P<0.05$ were considered statistically significant.

\section{RESULTS}

\section{NDGA-P21 Inhibits GBM Cell Proliferation}

NDGA is a natural product, which has been reported to inhibit growth of multiple tumors, such as breast cancer, cervical cancer, gastric cancer, lung cancer, prostate cancer, and neuroblastoma. ${ }^{11-18}$ In previous studies, we found Nordy, as a synthetic chiral derivative of NDGA, repressed the growth of glioma cells both in vitro and in vivo. ${ }^{7,19-21}$
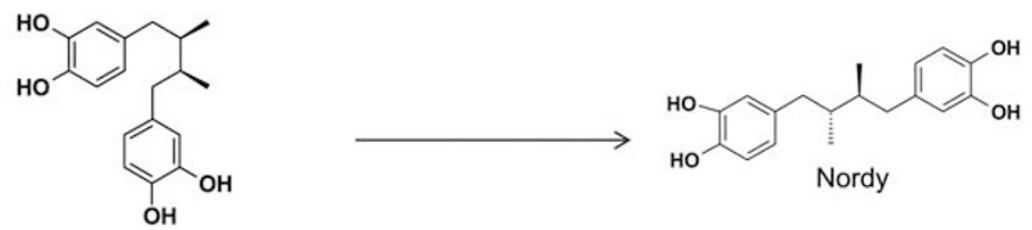

Nordihydroguaiaretic acid

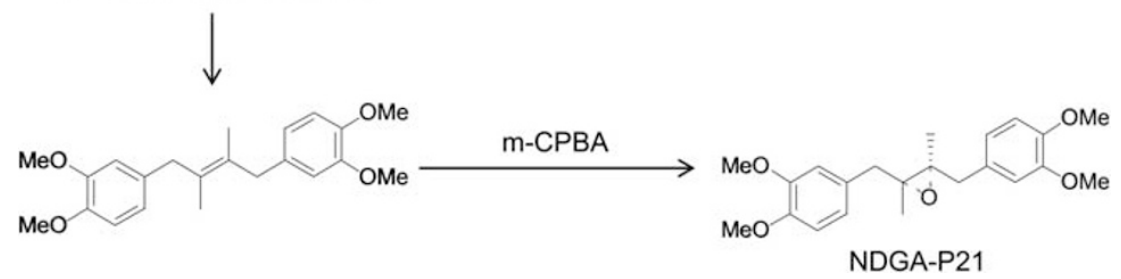

Figure 1 NDGA-P21 is synthesized based on the structure of nordihydroguaiaretic acid (NDGA), from the substrate (E)-4, 4'-(2, 3-dimethylbut-2-ene-1, 4-diyl) bis (1, 2-dimethoxybenzene). Both Nordy and NDGA-P21 are synthetized based on the structure of NDGA. NDGA-P21 came from the substrate (E)-4,4'-(2,3-dimethylbut-2-ene-1,4-diyl) bis (1, 2-dimethoxybenzene), and react with m-CPBA. 

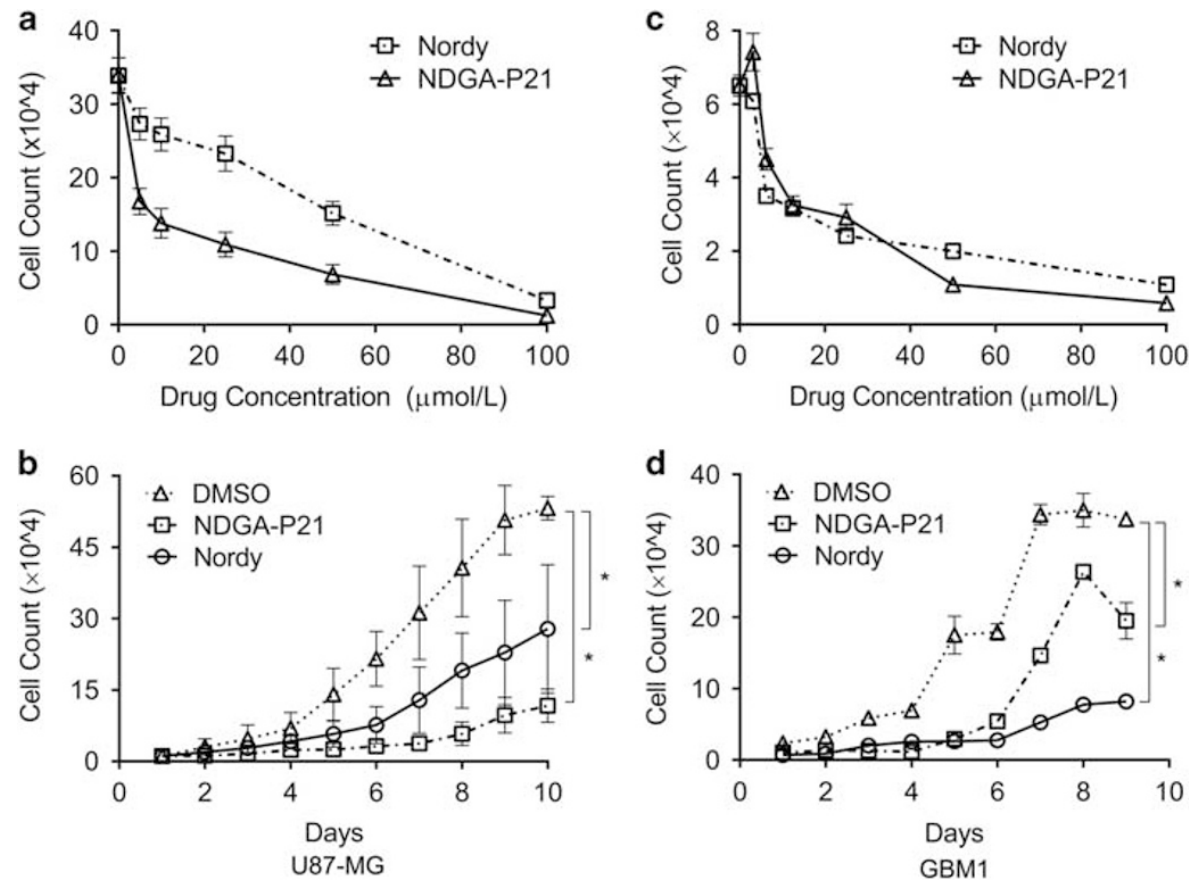

Figure 2 Nordihydroguaiaretic acid (NDGA)-P21 and Nordy inhibit glioma cell proliferation. (a) U87-MG cells were starved for 12 h, treated at different concentration of NDGA-P21, and Nordy for 3 days. IC 50 of NDGA-P21 and Nordy were: 7.43 and $34.52 \mu \mathrm{mol} / \mathrm{l}$. (b) U87-MG cells were treated with $50 \mu \mathrm{mol} / \mathrm{I}$ NDGA-P21, $50 \mu \mathrm{mol} / \mathrm{I}$ Nordy and 0.5\% DMSO, respectively, until day 11 . (c) GBM1 cells were treated as panel (a), IC 50 of NDGA-P21 and Nordy were 47.56 and $41.91 \mu \mathrm{mol} / \mathrm{l}$. (d) GBM1 cells were treated as panel (b). Here, P21 refers to NDGA-P21. DMSO-treated cells was used as control group. Data are presented as the mean \pm s.e.m. of three independent experiments carried out in quadruplicate. ${ }^{*} P<0.05$.

Based on NDGA structure, NDGA-P21, as a newly synthetic compound from the substrate (E)-4, 4'-(2, 3-dimethylbut-2ene-1, 4-diyl) bis (1, 2-dimethoxybenzene; Figure 1), showed stronger inhibitory effects on the growth of GBM cells than Nordy. We treated U87-MG cells and primary GBM cells with NDGA-P21 and Nordy for 3 days at the concentration of 0 , $10,25,50,100 \mu \mathrm{mol} / \mathrm{l}$, calculated their $\mathrm{IC}_{50}$ values, and found that for U87-MG cell line, IC $_{50}$ values of NDGA-P21, and Nordy were 7.43 and $34.52 \mu \mathrm{mol} / \mathrm{l}$ respectively (Figure 2a), and for primary GBM cells, $\mathrm{IC}_{50}$ values were 47.56 and $41.91 \mu \mathrm{mol} / \mathrm{l}$ respectively (Figure $2 \mathrm{~b}$ ). During administration, NDGA-P21-treated U87-MG cells began to show significant difference from DMSO-treated control group since day 7 until day 10 when cell proliferation reached a plateau. While Nordy-treated group began to show growth difference since day 8 , the inhibition by Nordy on U87-MG proliferation was not as strong as NDGA-P21 (Figure 2c). In the proneural-like primary GBM cells, Nordy exhibited stronger inhibition than NDGA-P21 (Figure 2d). Meanwhile, we examined the effects of NDGA-P21 and Nordy on the proliferation of a normal glial cell line HEB at the concentration of $50 \mu \mathrm{mol} / \mathrm{l}$, and found no significant difference among NDGA-P21, Nordy and DMSO group (Supplementary Data 1). As compared to Nordy, NDGA-P21 had stronger inhibitory effects on the proliferation of U87-MG cells, and similar effects in the primary GBM cells of proneural-like subtype.

\section{NDGA-P21 Arrests Cell Cycle of Glioma Cells, but with Little Effect On Apoptosis}

Previous studies of NDGA and Nordy suggest that cell cycle arrest, especially G1/S phase arrest, might be one of the underlying mechanisms for NDGA and its analogs to prevent cell proliferation in multiple tumors. ${ }^{8,11,12,14,22}$ To determine whether cell cycle arrest was involved in the growth inhibition by NDGA-P21, we applied flow cytometry analysis on the NDGA-P21 treated U87-MG cell to examine their cell cycle status by ModFit software. As compared to DMSO-treated group, cells in G0/G1 phase increased by $19.46 \%$ after NDGA-P21 treatment, whereas cells in S phase decreased by $17.82 \%$ (Figure 3a). Cell cycle of primary GBM cells after treatment showed similar effects. With NDGA-P21 treatment, primary GBM cells in G0/G1 phase increased by $12.16 \%$ whereas cells in S phase decreased by $13.77 \%$ (Figure $3 \mathrm{~b}$ ). To further explorer the underlying mechanisms, we examined the mRNA expressions of several G1/S and G2/M phase cell cycle hallmarks and inhibitors. In consistent with flow cytometry analysis, qPCR data revealed a significant decrease in the mRNA expressions of CCNE1, cyclin-dependent kinase 2 (CDK2) and CDK6 after NDGA-P21 treatment for $24 \mathrm{~h}$ (Figure 3c). The mRNA expressions of several G2/M phase checkpoints such as CDK1, CDC25c and CCNB1 were also decreased whereas the mRNA expression of CCNB1 inhibitor GADD45A was increased after NDGA-P21 and Nordy 
a
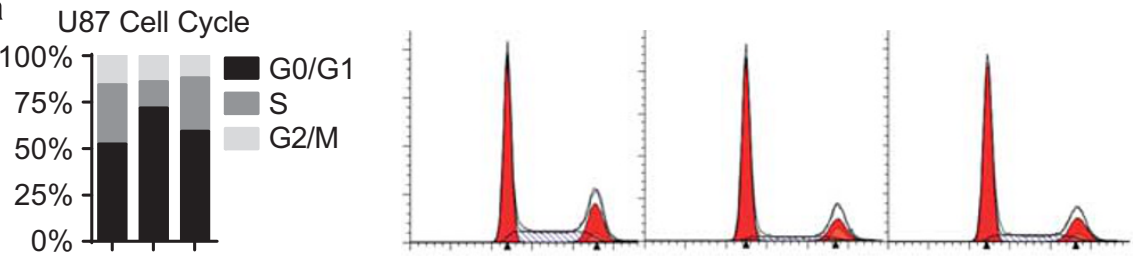

b GBM1 cell cycle
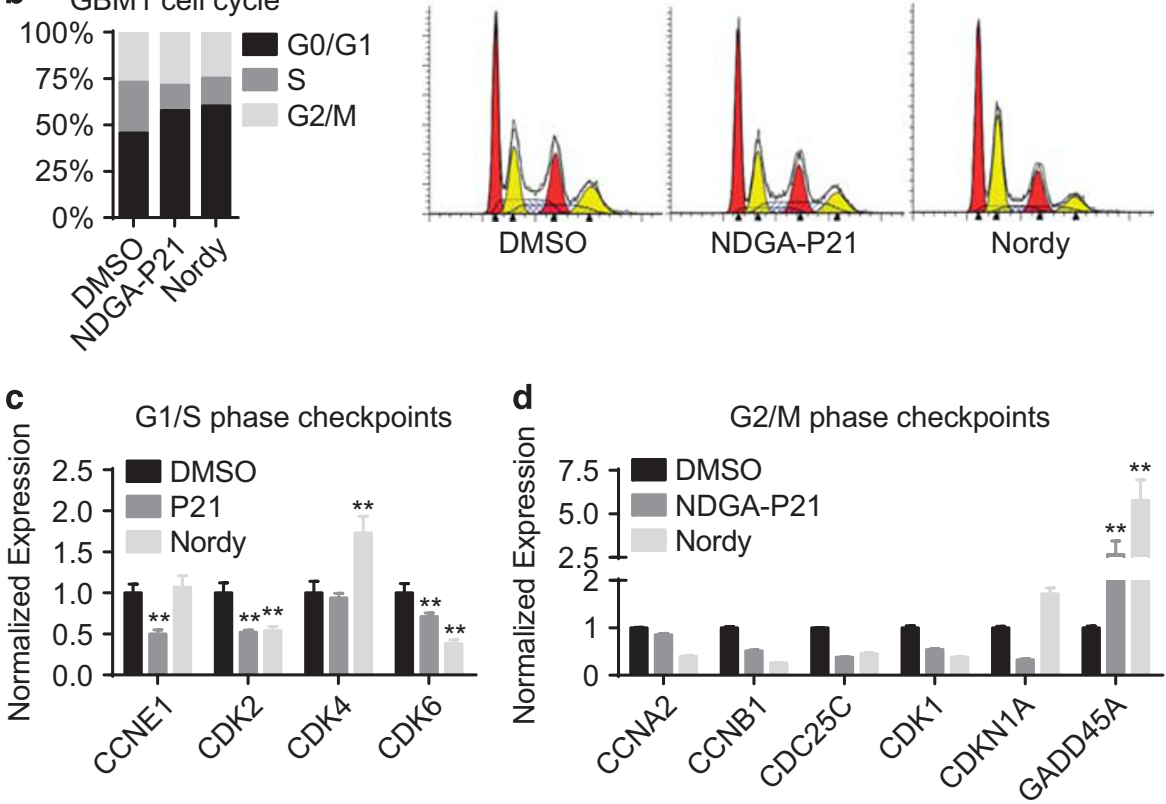

Figure 3 NDGA-P21 induces G1-to-S phase arrest in glioma cells. (a) Statistical analysis of cell cycle distribution tested with flow cytometer, U87-MG cells were pre-treated with $25 \mu \mathrm{mol} / \mathrm{I} \mathrm{NDGA-P21}$, Nordy or $0.25 \%$ DMSO. (b) Statistical analysis of cell cycle distribution tested with flow cytometer, GBM1 cells were pre-treated with $25 \mu \mathrm{mol} / \mathrm{I}$ NDGA-P21, Nordy or $0.25 \%$ DMSO. Three independent experiments were performed, and one representative data were exhibited. (c and d) Quantitative real-time PCR test for U87-MG cells were pre-treated with 25 umol/I NDGA-P21, Nordy or $0.25 \%$ DMSO for $24 \mathrm{~h}$. The column figure was the normalized mRNA expression as a fold change of the relevant GAPDH expression. Data are presented as the mean \pm s.e.m. of three independent experiments carried out in triplicate. ${ }^{*} P<0.05,{ }^{* *} P<0.01$.

treatment (Figure 3d). These results suggest that NDGA-P21 or Nordy treatment arrests the cell cycle of glioma cells in G1 phase.

Apoptosis is also suggested for NDGA inhibition of tumor cell proliferation. ${ }^{18}$ Thus, we also examined U87-MG apoptosis after NDGA-P21 and Nordy treatment. We found that treatment by NDGA-P21 or Nordy had no significant influence on the apoptosis of U87-MG cells (Supplementary Data 2).

\section{NDGA-P21 Inhibits GBM Cells Self-Renewal and Induces Differentiation}

Cancer stem-like cells or tumor-initiating cells in tumor have been thought as a key reason for tumor survival and progression. ${ }^{10,23}$ Self-renewal capability of GSLCs facilitates glioma cells to process self-copy, malignance, and differentiation into other cell types. ${ }^{24,25}$ We performed both colony formation and sphere formation assays to determine whether NDGA-P21 exerts any effects on the self-renewal of GSLCs. As shown in Figure 4, 9\% of U87-MG cells in soft agar with DMSO formed colonies after culture for 14 days, while only
$1 \%$ of the cells in the NDGA-P21-contained soft agar were able to into colonies, as compared to $3 \%$ in the presence of Nordy (Figure 4a). Colony formation of GBM1 was also decreased with NDGA-P21 and Nordy (Figure 4b). Akin to colony formation, sphere formation was significantly decreased in NDGA-P21 and Nordy-treated groups (Figure $4 \mathrm{c}$ and d). We also tested stemness markers of different drugs treated GSLCs, and observed an obviously decrease of Nanog mRNA expression in NDGA-P21 treated group (Figure 4e). GFAP is a protein usually expressed in glial cells and a differentiation marker of GSLC. ${ }^{9,10}$ No matter for U87-MG or GBM1 cells, GFAP level of GSLCs after treatment of NDGAP21 or Nordy was higher than control group, indicating that NDGA-P21 and Nordy promote differentiation of GSLCs (Figure $5 \mathrm{a}$ and $\mathrm{b}$ ). Thus, NDGA-P21 could significantly suppress stemness and promote differentiation in GSLCs.

\section{DISCUSSION}

NDGA has been known as a potential medicine to overcome oxidization, angiogenesis, neurological disorders and virus associated infection. ${ }^{3-6,26,27}$ Here, based on structure of 


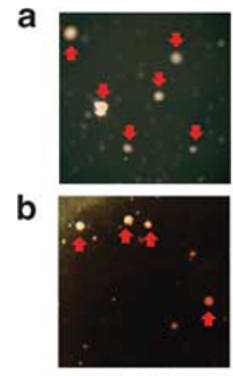

DMSO
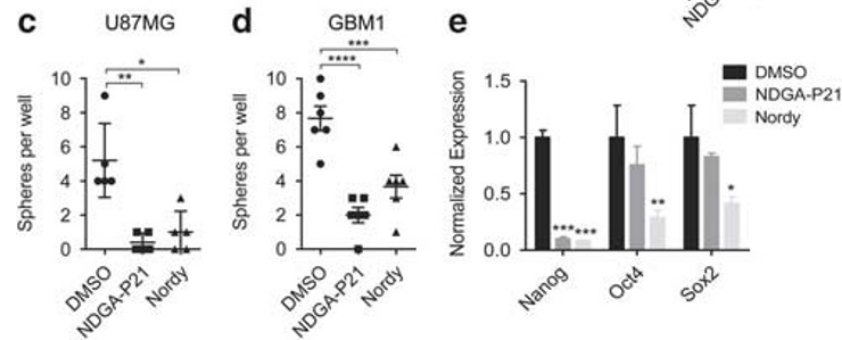

Figure 4 NDGA-P21 inhibits GBM cells self-renewal ability. (a) Bright field images of U87-MG colonies, under a stereo microscope. Cells were seeded in soft-agar containing NDGA-P21, Nordy or DMSO. Colonies with more than 50 cells were counted under a $\times 100$ inverted microscope. Each well was counted at 10 fields. (b) Bright field images of GBM1 colonies, under a digital camera. Cells were seeded in soft-agar containing NDGA-P21, Nordy, or DMSO. Colonies with more than 50 cells were counted under a $\times 100$ inverted microscope. Each well was counted at 10 fields. (c and d) U87-MG cells and GBM1 cells were maintained in neural stem cell culture medium for 14 days, with $25 \mu \mathrm{mol} / \mathrm{I}$ NDGA-P21, or $25 \mu \mathrm{mol} / \mathrm{I}$ Nordy. (e) Real-time qPCR test for stemness markers: Nanog, Oct4 and Sox2. U87-MG sphere cells were treated with $25 \mu \mathrm{mol} / \mathrm{l}$ NDGA-P21, $25 \mu \mathrm{mol} / \mathrm{l}$ Nordy, or $0.25 \%$ o DMSO for $24 \mathrm{~h}$ before test. All statistical data are presented as the mean \pm s.e.m. of three independent experiments carried out in triplicate. ${ }^{*} P<0.05,{ }^{* *} P<0.01,{ }^{* * *} P<0.001$, **** $P<0.0001$.

NDGA, we synthesized NDGA-P21, which exerted extensive killing effects on glioma cells. NDGA-P21 can significantly inhibit the growth of glioma cells as similar as Nordy, while the inhibitory effects of NDGA-P21 on U87-MG cells appeared earlier and stronger than Nordy. Furthermore, NDGA-P21, similar to Nordy, can suppress stemness and promote differentiation of GSLC.

In general, phenolic groups are thought to be the key functional factors for modification of NDGA. ${ }^{28}$ In our study, we changed the carbon chain of NDGA to yield NDGA-P21. And this novel compound showed strong inhibition effects of glioma cells. As there are many factors affecting cell growth, we focused on cell cycle and apoptosis as the potential mechanisms of NDGA-P21. By flow analysis of compoundstreated glioma cells, we found that there were more cells arrested in G1 phase but less in S phase after NDGA-P21 treatment. Then we analyzed mRNA expressions of several key cell cycle regulators. G1/S-specific cyclin-E1 (CCNE1), which accumulates at the G1-to-S phase boundary and is degraded as cells progress through $\mathrm{S}$ phase, ${ }^{29,30}$ forms a complex with $\mathrm{CDK} 2$, and functions as a regulatory factor of a
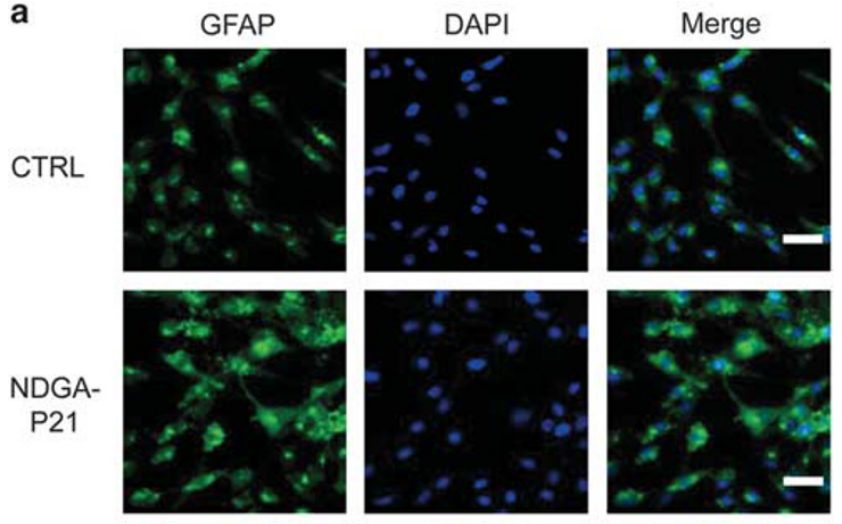

Nordy
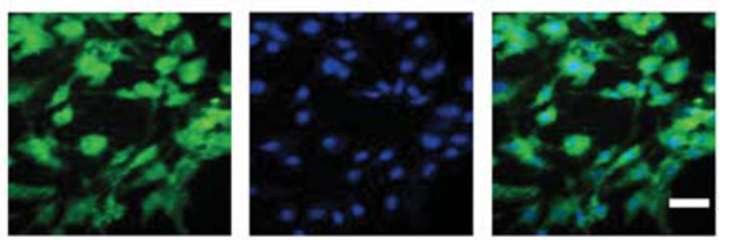

b
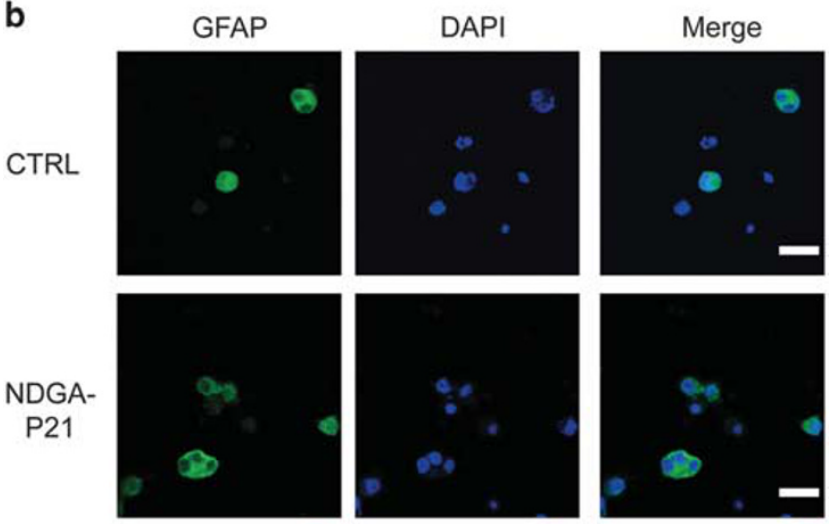

Nordy
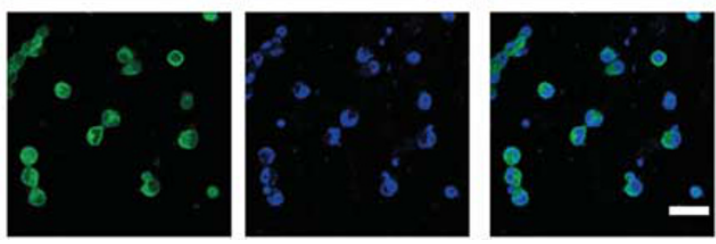

Figure 5 NDGA-P21 induces differentiation of GSLCs. (a) Immunofluorescence showing GFAP protein (green) in U87-MG GSLCs elicited by $25 \mu \mathrm{mol} / \mathrm{I} \mathrm{NDGA-P21}$ or Nordy treatment. (b) Immunofluorescence showing GFAP protein (green) in GBM1 GSLCs elicited by $25 \mu \mathrm{mol} / \mathrm{l}$ NDGA-P21 or Nordy treatment. Cell nucleuses were stained blue with DAPI. Scale bars of the figures showed as $50 \mu \mathrm{m}$ in real distance. $0.25 \%$ DMSO-treated GSLCs were control group, labeled as CTRL.

CDK2 activity. ${ }^{31,32}$ We found that both NDGA-P21 and Nordy were able to repress the mRNA expression of CCNE1 and CDK2. CDK6, as another important regulator during G1-to-S phase transition, was also downregulated with NDGA-P21 and Nordy. Importantly, CDK6 also has a specific oncogenic capability. ${ }^{33,34}$ Suppression of CDK6 expression refers to another potential mechanism for NDGA-P21 to suppress tumor growth. Meanwhile, cyclin B1 (CCNB1) and CDK1 were suppressed by NDGA-P21, and 
CCNB1 inhibitor GADD45A was strongly upregulated, indicating that G2-to-M phase arrest was also induced. Thus, NDGA-P21 arrested cell cycle of glioma cells. Apoptosis has been suggested as one of the mechanisms underlying the inhibition of NDGA and its derivatives in multiple tumors. ${ }^{4,16,17,35,36}$ However, we found that similar to Nordy, NDGA-P21 had little effects on apoptosis. The results suggest different structure modification of NDGA leads an alternative mechanism for inhibition of tumor cells.

Glioma-stem-like cells are defined as glioma cells that retain extensive self-renewal potential through multiple generations, and are also known as a limited subpopulation of tumor-initiating cells. ${ }^{37}$ GSLCs are thought to be the responsible cell population for glioma invasion, migration, progression, and recurrence. ${ }^{38-41}$ Neurospheres and orospheres are used for propagation and enrichment of CSCs, including GSLCs. ${ }^{42,43}$ Orospheres are non-adherent tumor cells that may be cultured in soft agar to colonies and retained stemness and self-renewal capability. In this study, we found NDGA-P21 and Nordy could suppress GSLC self-renewal and promote GSLC differentiation, which mainly owns to Nanog, a pluripotency sustaining transcription factor. Physiologically, Nanog only expresses by germline stem cells and during very early embryonic stages. ${ }^{44}$ Nanog activates tumor suppressor P53 and facilitates proliferation of stem cells, but in the absence of P53, Nanog is overexpressed in a variety of human cancer types such as glioma. ${ }^{45}$ Our study reveals that NDGAP21 suppresses Nanog expression and inhibits self-renewal of GSLCs, suggesting that this compound could suppress tumorigenic capability of glioma.

NDGA-P21 has the potential therapeutic effects on glioma, not only because NDGA-P21 inhibits proliferation of glioma cells directly, but also because it inhibits the progression of most malignant groups of glioma. However, NDGA-P21 showed limit water solubility, and that might be an obstacle for further test for this molecule. So we are trying to modify this molecule for better solubility. Recently, NDGA and its analog terameprocol finished phase I/II clinical trials as anticancer agents, and showed limited severe adverse effects, ${ }^{15,46,47}$ indicating that this family of small molecules has great potential on clinic treatment. Our novel NDGA-based small molecular compound strongly inhibited proliferation of glioma cells, suppressed self-renewal, and induced differentiation of glioma stem-like cells, at least in vitro, predicting the therapeutic application for glioma. Although our current study illustrated NDGA-P21 inhibition to GBM cell proliferation in several aspects in vitro, we still know little about the real effects after NDGA-P21 administration in vivo. Moreover, the underlying mechanisms involved in the suppression of multiple tumors by NDGA and its derivatives still need further exploration in the future.

Supplementary Information accompanies the paper on the Laboratory Investigation website (http://www.laboratoryinvestigation.org).
ACKNOWLEDGMENTS

This work was supported by the National Key Research And Development Program (2016YFA0101200) and the National Natural Science Foundation of China (NSFC No. 30930103).

\section{DISCLOSURE/CONFLICT OF INTEREST}

The authors declare no conflict of interest.

1. Chen W, Zheng R, Baade PD, et al. Cancer statistics in China, 2015. CA Cancer J Clin 2016;66:115-132.

2. Siegel RL, Miller KD, Jemal A. Cancer statistics, 2016. CA Cancer J Clin 2016;66:7-30.

3. Arteaga $S$, Andrade-Cetto A, Cardenas R. Larrea tridentata (Creosote bush), an abundant plant of Mexican and US-American deserts and its metabolite nordihydroguaiaretic acid. J Ethnopharmacol 2005;98: 231-239.

4. Hernandez-Damian J, Anderica-Romero AC, Pedraza-Chaverri J. Paradoxical cellular effects and biological role of the multifaceted compound nordihydroguaiaretic acid. Arch Pharm 2014;347:685-697.

5. Lu JM, Nurko J, Weakley SM, et al. Molecular mechanisms and clinical applications of nordihydroguaiaretic acid (NDGA) and its derivatives: an update. Med Sci Monit 2010;16:Ra93-100.

6. Chen Q. Nordihydroguaiaretic acid analogues: their chemical synthesis and biological activities. Curr Top Med Chem 2009;9:1636-1659.

7. Chen JH, Bian XW, Yao XH, et al. Nordy, a synthetic lipoxygenase inhibitor, inhibits the expression of formylpeptide receptor and induces differentiation of malignant glioma cells. Biochem Biophys Res Commun 2006;342:1368-1374.

8. Zhao J, Zhao Y, Chen W, et al. The differentiation-inducing effect of Nordy on HPV-16 subgenes-immortalized human endocervical cells H8. Anticancer Drugs 2008;19:713-719.

9. Wang B, Yu SC, Jiang JY, et al. An inhibitor of arachidonate 5-lipoxygenase, Nordy, induces differentiation and inhibits selfrenewal of glioma stem-like cells. Stem Cell Rev 2011;7:458-470.

10. Wang Z, Wang B, Shi $Y$, et al. Oncogenic miR-20a and miR-106a enhance the invasiveness of human glioma stem cells by directly targeting TIMP-2. Oncogene 2015;34:1407-1419.

11. Zavodovskaya M, Campbell MJ, Maddux BA, et al. Nordihydroguaiaretic acid (NDGA), an inhibitor of the HER2 and IGF-1 receptor tyrosine kinases, blocks the growth of HER2-overexpressing human breast cancer cells. J Cell Biochem 2008;103:624-635.

12. Gao P, Zhai FEl, Guan LEl, et al. Nordihydroguaiaretic acid inhibits growth of cervical cancer SiHa cells by up-regulating p21. Oncol Lett 2011;2:123-128.

13. Ryan CJ, Zavodovskaya M, Youngren JF, et al. Inhibitory effects of nordihydroguaiaretic acid (NDGA) on the IGF-1 receptor and androgen dependent growth of LAPC-4 prostate cancer cells. Prostate 2008;68: $1232-1240$

14. Ozkan EE. Plasma and tissue insulin-like growth factor-I receptor (IGF-IR) as a prognostic marker for prostate cancer and anti-IGF-IR agents as novel therapeutic strategy for refractory cases: a review. Mol Cell Endocrinol 2011;344:1-24.

15. Friedlander TW, Weinberg VK, Huang $Y$, et al. A phase II study of insulin-like growth factor receptor inhibition with nordihydroguaiaretic acid in men with non-metastatic hormone-sensitive prostate cancer. Oncol Rep 2012;27:3-9.

16. Xu C, Li W, Qiu P, et al. The therapeutic potential of a novel non-ATPcompetitive fibroblast growth factor receptor 1 inhibitor on gastric cancer. Anticancer Drugs 2015;26:379-387.

17. Wu J, Wei $T$, Tang $Q$, et al. Discovery and anti-cancer evaluation of two novel non-ATP-competitive FGFR1 inhibitors in non-small-cell lung cancer. BMC Cancer 2015;15:276.

18. Meyer GE, Chesler L, Liu D, et al. Nordihydroguaiaretic acid inhibits insulin-like growth factor signaling, growth, and survival in human neuroblastoma cells. J Cell Biochem 2007;102:1529-1541.

19. Bian XW, Xu JP, Ping YF, et al. Unique proteomic features induced by a potential antiglioma agent, Nordy (dl-nordihydroguaiaretic acid), in glioma cells. Proteomics 2008;8:484-494.

20. Ping $\mathrm{YF}, \mathrm{Yao} \mathrm{XH}, \mathrm{Chen} \mathrm{JH}$, et al. The anti-cancer compound Nordy inhibits CXCR4-mediated production of IL-8 and VEGF by malignant human glioma cells. J Neurooncol 2007;84:21-29. 
21. Chen $\mathrm{JH}$, Yao $\mathrm{XH}$, Gong W, et al. A novel lipoxygenase inhibitor Nordy attenuates malignant human glioma cell responses to chemotactic and growth stimulating factors. J Neurooncol 2007;84:223-231.

22. Davies C, Hogarth LA, Mackenzie KL, et al. p21(WAF1) modulates drug-induced apoptosis and cell cycle arrest in B-cell precursor acute lymphoblastic leukemia. Cell Cycle 2015;14:3602-3612.

23. Suva ML, Rheinbay $\mathrm{E}$, Gillespie SM, et al. Reconstructing and reprogramming the tumor-propagating potential of glioblastoma stem-like cells. Cell 2014;157:580-594.

24. Wang R, Chadalavada K, Wilshire J, et al. Glioblastoma stem-like cells give rise to tumour endothelium. Nature 2010;468:829-833.

25. Lama G, Mangiola A, Proietti G, et al. Progenitor/stem cell markers in brain adjacent to glioblastoma: GD3 ganglioside and NG2 proteoglycan expression. J Neuropathol Exp Neurol 2016;75:134-147.

26. Yamada M, Ono K, Hamaguchi T, et al. Natural phenolic compounds as therapeutic and preventive agents for cerebral amyloidosis. Adv Exp Med Biol 2015;863:79-94.

27. Soto-Acosta R, Bautista-Carbajal P, Syed GH, et al. Nordihydroguaiaretic acid (NDGA) inhibits replication and viral morphogenesis of dengue virus. Antiviral Res 2014;109:132-140.

28. Remya C, Dileep KV, Tintu I, et al. In vitro inhibitory profile of NDGA against AChE and its in silico structural modifications based on ADME profile. J Mol Model 2013;19:1179-1194.

29. Parisi T, Beck AR, Rougier N, et al. Cyclins E1 and E2 are required for endoreplication in placental trophoblast giant cells. EMBO J 2003;22: 4794-4803.

30. Ohtsubo M, Theodoras AM, Schumacher J, et al. Human cyclin E, a nuclear protein essential for the G1-to-S phase transition. Mol Cell Biol 1995;15:2612-2624.

31. Koff A, Giordano A, Desai D, et al. Formation and activation of a cyclin E-cdk2 complex during the G1 phase of the human cell cycle. Science 1992;257:1689-1694.

32. Honda R, Lowe ED, Dubinina $E$, et al. The structure of cyclin E1/CDK2: implications for CDK2 activation and CDK2-independent roles. EMBO J 2005;24:452-463.

33. Tadesse $S$, Yu M, Kumarasiri M, et al. Targeting CDK6 in cancer: state of the art and new insights. Cell Cycle 2015;14:3220-3230.

34. Bellail AC, Olson JJ, Hao C. SUMO1 modification stabilizes CDK6 protein and drives the cell cycle and glioblastoma progression. Nat Commun 2014;5:4234.
35. Gerhauser C. Cancer chemoprevention and nutriepigenetics: state of the art and future challenges. Top Curr Chem 2013;329:73-132.

36. Castro-Gamero AM, Borges KS, Moreno DA, et al. Tetra-O-methyl nordihydroguaiaretic acid, an inhibitor of Sp1-mediated survivin transcription, induces apoptosis and acts synergistically with chemo-radiotherapy in glioblastoma cells. Invest New Drugs 2013;31: 858-870.

37. Singh SK, Hawkins C, Clarke ID, et al. Identification of human brain tumour initiating cells. Nature 2004;432:396-401.

38. Ye XZ, Xu SL, Xin YH, et al. Tumor-associated microglia/macrophages enhance the invasion of glioma stem-like cells via TGF-beta1 signaling pathway. J Immunol 2012;189:444-453.

39. Hira VV, Ploegmakers KJ, Grevers F, et al. CD133+ and nestin+ glioma stem-like cells reside around CD31+ arterioles in niches that express SDF-1alpha, CXCR4, osteopontin and cathepsin K. J Histochem Cytochem 2015;63:481-493.

40. Calinescu AA, Yadav VN, Carballo $E$, et al. Survival and proliferation of neural progenitor derived glioblastomas under hypoxic stress is controlled by a CXCL12/CXCR4 autocrine positive feedback mechanism. Clin Cancer Res 2016;23:1250-1262.

41. Tate $C M$, Pallini $R$, Ricci-Vitiani $L$, et al. A BMP7 variant inhibits the tumorigenic potential of glioblastoma stem-like cells. Cell Death Differ 2012;19:1644-1654.

42. Yu SC, Ping YF, Yi L, et al. Isolation and characterization of cancer stem cells from a human glioblastoma cell line U87. Cancer Lett 2008;265: 124-134.

43. Krishnamurthy S, Nör JE. Orosphere assay: a method for propagation of head and neck cancer stem cells. Head Neck 2013;35:1015-1021.

44. Chambers I, Colby D, Robertson $\mathrm{M}$, et al. Functional expression cloning of nanog, a pluripotency sustaining factor in embryonic stem cells. Cell 2003;113:643-655.

45. Zbinden M, Duquet A, Lorente-Trigos A, et al. NANOG regulates glioma stem cells and is essential in vivo acting in a cross-functional network with GLI1 and p53. EMBO J 2010;29:2659-2674.

46. Grossman SA, Ye X, Peereboom D, et al. Phase I study of terameprocol in patients with recurrent high-grade glioma. Neuro Oncol 2012;14: 511-517.

47. Tibes R, McDonagh KT, Lekakis L, et al. Phase I study of the nove Cdc2/CDK1 and AKT inhibitor terameprocol in patients with advanced leukemias. Invest New Drugs 2015;33:389-396. 